\title{
Efeitos do estresse de trabalho sobre parâmetros seminais de cães da raça Rottweiler
}

Carlos de Almeida

BAPTISTA SOBRINHO ${ }^{1,2}$

Luciana Keiko HATAMOTO-

ZERVOUDAKIS ${ }^{3}$

Valquíria Hyppolito

BARNABE $^{1}$

Marcílio $\mathrm{NICHI}^{1}$

Claudio Alvarenga de

OLIVEIRA $^{1}$

\section{Correspondência para:}

Carlos de Almeida Baptista Sobrinho, Exército Brasileiro - $2^{\circ}$ Batalhão de Polícia do Exército-Seção de Cães de Guerra, Rua Raul Lessa 52, 06236-100, Osasco, São Paulo, cabsobrinho@uol.com.br / sobrinho@usp.br

Recebido para publicação: 17/11/2005 Aprovado para publicação: 30/04/2009

\author{
1 - Departamento de Reprodução Animal da Faculdade de Medicina \\ Veterinária e Zootecnia da Universidade de São Paulo, São Paulo-SP \\ 2 - Exército Brasileiro, $2^{\circ}$ Batalhão de Polícia do Exército - Seção de Cães de \\ Guerra, Osasco-São Paulo \\ 3 - Faculdade de Agronomia e Medicina Veterinária da Universidade Federal \\ do Mato Grosso, Cuiabá-MT
}

\section{Resumo}

Palavras-chave: Sêmen.

O presente trabalho teve como objetivo avaliar os efeitos do estresse de trabalho sobre a fertilidade de cães machos. Foram utilizados 18 cães da raça rottweiler, férteis, com idade média de 4 anos, distribuídos aleatoriamente em dois grupos: repouso (controle) e trabalho (tratamento). O tratamento era composto por 5 fases: 1-adaptação, 2adestramento básico, 3-adestramento militar e condicionamento, 4acampamento, 5-repouso. Durante todo o período experimental, foram feitas duas coletas de sêmen semanais, para avaliação do ejaculado. No final de cada fase foi realizada coleta de sangue para dosagem dos níveis plasmáticos de cortisol. Os dados foram analisados pelo SAS, á= 5\%. Observou-se efeito negativo do estresse sobre os parâmetros motilidade (72,63 vs. 57,62, p<0,0001) e vigor espermático (3,06 vs. 2,52, $\mathrm{p}<0,0001)$, porcentagem de defeitos maiores (16,01 vs. $26,80, \mathrm{p}<0,0001)$ e totais $(29,61$ vs. $40,34, \mathrm{p}<0,0001)$. Observou-se interação $(\mathrm{p}<0,0001)$ entre tempo e tratamento sobre a variável cortisol, indicando que o tipo de agente estressante (fase) interfere no nível plasmático de cortisol. Com base nesses resultados, é possível concluir que o estresse de trabalho interferiu de maneira negativa na fertilidade dos cães.

\section{Introdução}

Nos últimos anos, a inserção social dos animais domésticos tem crescido significativamente. Além de animais de companhia ${ }^{1}$, os cães desempenham várias funções: servem como guias de deficientes visuais, auxiliam no tratamento de algumas doenças, pastoreiam rebanhos, auxiliam na caça, atuam como guarda de bens e/ou pessoas, e são utilizados para a detecção de drogas em aeroportos e em atividades policiais e militares. Devido a esta maior proximidade com o ser humano os cães tornam-se mais susceptíveis a comportamentos e alterações de saúde que antes eram exclusivos do homem, como o sedentarismo, a obesidade e o estresse.

O estresse é a incapacidade de um animal interagir com o ambiente em igualdade de condições ${ }^{2}$ sendo que, diferentes autores apontam a correlação positiva existente entre o estresse e a reprodução ${ }^{2,3,4}$. O estresse funcional e o estresse de trabalho têm grande influência na qualidade do sêmen, e prejudicam a fertilidade em humanos. ${ }^{5}$ A principal manifestação do estresse nos machos é a significativa queda de qualidade do sêmen, expressa pela redução da motilidade espermática $^{3,6}$ pelo aumento do número de espermatozóides mortos, pelo aumento das alterações na membrana espermática ${ }^{6}$, pelo elevado grau de retenção de gota citoplasmática e pelas alterações acrossomais ${ }^{3}$ que indicam má função epididimária.

Desta forma, o conhecimento do mecanismo pelo qual o estresse pode afetar 
a qualidade seminal, e do seu impacto na fertilidade dos cães, pode levar a propostas que melhorem a eficácia no manejo dos cães de trabalho, melhorando a sua qualidade de vida.

\section{Material e Método}

O presente experimento foi realizado no canil da Seção de Cães-de-Guerra do $2^{\circ}$ Batalhão de Polícia do Exército (SecCG/ $2^{\circ} \mathrm{BPE}$, Figura 1), localizado no município de Osasco (SP), no período de março a julho de 2001. Foram utilizados dezoito cães machos da raça rottweiler, do canil da SecCG $/ 2^{\circ} \mathrm{BPE}$, com idade média de quatro anos e peso médio de $40 \mathrm{~kg}$. Antes do início do experimento os animais foram vermifugados, vacinados, e submetidos a testes para a detecção de brucelose e de leptospirose no Laboratório do Departamento de Medicina Veterinária Preventiva da Faculdade de Medicina Veterinária e Zootecnia da Universidade de São Paulo. Somente os animais negativos para essas doenças participaram da investigação.

Os animais foram alojados em boxes individuais de doze metros quadrados. Recebiam ração comercial duas vezes ao dia, de modo a suprir os requerimentos nutricionais preconizados pelo National Research Council ${ }^{7}$, e água ad libitum. Diariamente eram examinados na enfermaria veterinária da SecCG $/ 2^{\circ} \mathrm{BPE}$, e a cada dez dias eram submetidos à pesagem. Os animais foram divididos aleatoriamente em dois grupos: um grupo de animais envolvidos no trabalho de adestramento (grupo tratamento, GT); o outro mantido em repouso (grupo controle, GC).

$\mathrm{O}$ adestramento e treinamento militar consistiram de uma rotina diária de exercícios de obediência básica, exercícios de adestramento militar e condicionamento físico. O experimento foi composto por cinco fases: 1. Adaptação dos cães ao canil e ao manejo; 2. Exercícios de adestramento básico; 3. Condicionamento físico e exercícios de adestramento, 4. Exercícios no acampamento (fase de maior trabalho e desgaste físico) e, 5. Período de recuperação dos cães.

O estresse foi caracterizado através da dosagem de cortisol plasmático ${ }^{8}$ e pelo escore corporal ${ }^{1}$. Amostras de $5 \mathrm{~mL}$ de sangue foram colhidas por venopunção cefálica radial. Em seguida, o plasma foi separado por centrifugação a $2500 \mathrm{~g}$ durante dez minutos e armazenado congelado $\left(-8^{\circ} \mathrm{C}\right)$ até o momento da análise. Ao final de cada período foram feitas 6 coletas seriadas de sangue ${ }^{9}$ às $10 \mathrm{~h} 00,14 \mathrm{~h} 00,18 \mathrm{~h} 00,22 \mathrm{~h} 00$, 02h00 e 06h00 horas. A determinação dos níveis plasmáticos de cortisol foi feita no Laboratório de Dosagens Hormonais (LDH) do Departamento de Reprodução Animal da Faculdade de Medicina Veterinária

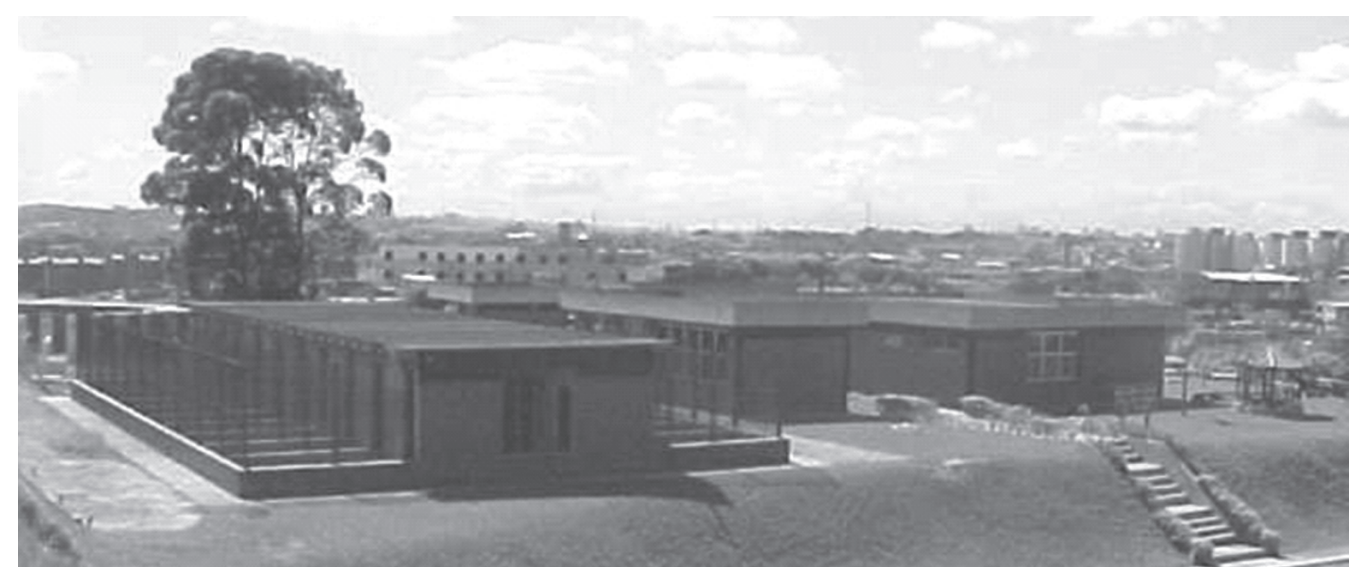

Figura 1 - Vista da Seção de Cães-de-Guerra do $2^{\circ}$ BPE, tendo o pavilhão de canis individuais à esquerda 
e Zootecnia da Universidade de São Paulo (VRA/FMVZ-USP). As dosagens foram realizadas com kits comerciais de radioimunoensaio (Coat-a-Count Cortisol ${ }^{\circledR}$, DPC Lab.).

O escore corporal e a consistência testicular foram avaliados antes de cada coleta de sêmen. Para a avaliação do escore corporal foi utilizada a metodologia de Hand et al. ${ }^{10}$, onde $1=$ muito magro; 2 = magro; $3=$ normal; $4=$ sobrepeso; $5=$ obeso. $A$ consistência testicular foi mensurada por meio de palpação sempre pelo mesmo técnico. Para efeito de classificação, foi utilizada uma escala de um a cinco, onde: 1 consistência friável; 2 - consistência intermediária; 3 - consistência firme (desejável); 4 - consistência túrgida e, 5 consistência dura.

Foram realizadas duas coletas de sêmen por semana, através de estimulação manual, com o animal em estação ${ }^{6,11,12}$. O sêmen foi colhido em tubo cônico graduado protegido contra choques físicos e luz, e mantido a $37^{\circ} \mathrm{C}^{12,13}$, sem a presença de fêmea no cio. Somente a segunda fração, rica em espermatozóides, foi analisada. Para minimizar o estresse, o mesmo enfermeiro veterinário realizou todas as coletas de sêmen.

Imediatamente após a coleta foi avaliado o volume da segunda fração do ejaculado diretamente no tubo de colheita ${ }^{6,14}$ e retiradas amostras para a avaliação da concentração espermática, motilidade progressiva, vigor espermático e da morfologia espermática ${ }^{6,11,12}$, além da aferição do $\mathrm{pH}^{6,11,12,14,15}$.

A morfologia espermática foi avaliada através de preparações em câmara úmida, avaliadas em aumento de 1000x e classificada segundo Oettlé e Soley ${ }^{16}$. As alterações encontradas foram agrupadas em defeitos maiores, defeitos menores e defeitos totais ${ }^{6,12,14}$.

Este foi um experimento inteiramente casualizado com dois tratamentos. Foram consideradas as variáveis: peso e escore corporal, consistência testicular, volume e pH da segunda fração do ejaculado, motilidade progressiva, vigor e morfologia espermáticas (defeitos maiores, menores e totais) e níveis plasmáticos de cortisol. Os resultados dessas variáveis foram analisados com o programa The SAS System for Windows V8 (SAS Institute Inc., Cary, NC, USA, 2000), com um nível de significância de 5\%.

As variáveis: escore corporal, consistência testicular, porcentagem de defeitos menores e níveis plasmáticos de cortisol não obedeceram à normalidade dos resíduos sendo, portanto, consideradas e analisadas como dados não paramétricos, e submetidas ao PROC NPAR1WAY e ao teste de Wilcoxon. As demais variáveis se comportaram como variáveis paramétricas e foram submetidas a ANOVA e ao teste Tukey. Também foi realizada a correlação de Pearson entre as variáveis paramétricas e ao Teste de Spearman entre as variáveis não paramétricas.

\section{Resultados e Discussão}

A análise de variância demonstrou efeitos estatisticamente significativos $(\mathrm{p}<0,0001)$ do tratamento, da coleta e da interação coleta $\mathrm{X}$ tratamento sobre os níveis de cortisol plasmático. Indicando que o estresse de trabalho, independente do tipo de trabalho (fases do tratamento), induz ao quadro de estresse (Tabela 1). Diferenças nas características estressantes e nas características individuais dos cães promovem respostas variáveis. ${ }^{8}$ Entretanto, todas elas promovem aguda elevação nos níveis plasmáticos de cortisol, razão pela qual ele é utilizado como indicador de quadros de estresse. ${ }^{8,17}$

$\mathrm{Na}$ presente investigação, os níveis plasmáticos médios de cortisol foram de $1,094 \pm 0,913 \mathrm{ng} / \mathrm{dL}$ para o grupo controle, e de $1,376 \pm 0,800 \mathrm{ng} / \mathrm{dL}$ para o grupo tratamento (Tabela 1).

Contrariando os achados de Camacho", que encontrou valores elevados à noite e valores decrescentes durante $\mathrm{O}$ período diurno, não foram observados variações diárias nos níveis plasmáticos de cortisol neste trabalho. Essa divergência de resultados pode ser devido aos animais 
Tabela 1 - Média e desvio padrão (Md \pm DP) do nível plasmático de cortisol (ng/dL) de cães militares da raça rottweiler submetidos ao estresse de treinamento (GT) e de cães em repouso (GC) em função da fase do treinamento ${ }^{1}-2001$

\begin{tabular}{cccc}
\hline \multirow{2}{*}{ Fase do Treinamento ${ }^{1}$} & \multicolumn{2}{c}{ Nível plasmático de cortisol (ng/dL) } & \multirow{2}{*}{$\mathrm{p}$} \\
\cline { 2 - 4 } & $\mathrm{GT}$ & $\mathrm{GC}$ & 0,1984 \\
2 & $1,36 \pm 0,62$ & $1,68 \pm 1,02$ & 0,6187 \\
3 & $1,61 \pm 1,17$ & $1,65 \pm 1,12$ & $<0,0001$ \\
4 & $1,33 \pm 0,57$ & $0,72 \pm 0,35$ & 0,0002 \\
5 & $1,43 \pm 0,76$ & $0,92 \pm 0,70$ & $<0,0001$ \\
\hline Média & $1,14 \pm 0,67$ & $0,49 \pm 0,029$ & 0,0047 \\
\hline Fase do Treinamento: & 1. Adaptação dos cães ao canil e ao manejo; 2. Exercícios de \\
adestramento básico; 3. Condicionamento fisico e exercícios de adestramento; 4. Exercícios no \\
acampamento (fase de maior trabalho e desgaste físico); 5. Período de repouso dos cães
\end{tabular}

utilizados, Camacho9 avaliou cães sem raça definida (SRD), de no máximo $20 \mathrm{~kg}$ e de ambos os sexos, enquanto que no presente experimento somente foram utilizados machos, da raça rottweiler, com peso médio de $40 \mathrm{~kg}$. De acordo com Encarnação ${ }^{2} \mathrm{o}$ peso e a raça são importantes fatores que interferem nos níveis plasmáticos de cortisol e corticosterona. Verificou-se correlação positiva entre os níveis séricos de cortisol e a consistência testicular $(r=0,215 ; \mathrm{p}=0,0421)$ e correlação negativa entre tais níveis e a concentração espermática $(r=-0,287$; $\mathrm{p}=0,0061)$. Essas correlações, apesar de serem consideradas baixas, são indicativas de que o estresse pode alterar a qualidade seminal através da diminuição da concentração e da diminuição da consistência testicular que são características observadas em animais com degeneração testicular. ${ }^{18}$

Este trabalho não observou redução do peso nem do escore corporal nos animais do GT, contrariando os achados de outros autores ${ }^{1,3,4}$, os quais afirmam que animais submetidos ao estresse apresentam, entre outras alterações fisiológicas, redução no consumo alimentar, no peso e no escore corporal. Uma possível explicação para o observado no presente estudo é a carga intensa de exercícios físicos a que foram submetidos os animais do GT, com possível ganho de massa muscular, o que acabaria por mascarar o efeito deletério do estresse sobre o peso e o escore corporal.

$O$ estado nutricional tem relação direta com os parâmetros seminais, e pode ser avaliado pelo peso vivo e pelo escore corporal $^{1}$. Os resultados encontrados, de peso e de escore corporal, demonstram que não houve diferenças entre o grupo tratado e o grupo controle. Assim, pode-se afirmar que o estado nutricional dos cães foi controlado durante o período experimental, indicando que as alterações seminais encontradas foram decorrentes apenas do estresse.

O estresse não alterou significantemente $(p>0,05)$ a consistência testicular contrariando a literatura consultada ${ }^{19}$. Todavia observaram-se correlações estatísticas entre a consistência testicular e a motilidade espermática $(\mathrm{r}=0,225 ; \mathrm{p}=0,0379)$, os níveis plasmáticos de cortisol $(\mathrm{r}=0,357 ; \mathrm{p}=0,0008)$, o pH $(\mathrm{r}=-0,282 ; \mathrm{p}=0,0090)$, a concentração espermática $(\mathrm{r}=-0,261 ; \mathrm{p}=0,0158)$ e os defeitos menores $(r=-0,231 ; p=0,0375)$. Indicando que a consistência testicular está relacionada com diferentes indicadores de fertilidade. ${ }^{3,4,5,12,18}$

O método da manipulação digital mostrou-se eficiente para a obtenção do sêmen canino, e de fácil aceitação pelos animais. A ausência de fêmea no cio durante os períodos de condicionamento e experimental não interferiu na obtenção do sêmen, conforme já haviam observado 
outros pesquisadores. ${ }^{6,12,14}$

O estresse não influenciou o volume da $2^{\mathrm{a}}$ fração do ejaculado uma vez que os valores encontrados não diferiram $(p>0,05)$ entre os animais GT e GC além de apresentarem-se semelhante aos valores encontrados por Seager e Platz ${ }^{15}$, porém superiores aos resultados de Leite $\mathrm{Netto}^{14} \mathrm{e}$ inferiores aos achados de Souza et al. ${ }^{12}$. Tais diferenças podem estar relacionadas às raças estudadas e à freqüência das coletas, uma vez que nenhum desses autores avaliou ejaculados de cães da raça Rottweiler.

A motilidade espermática apresentada pelos animais do GC (Tabela 2) assemelha-se aos valores obtidos por outros autores ${ }^{12,14}$, que utilizaram cães de raças e portes diferentes daqueles empregados nesta investigação; assim, pode-se afirmar que a variável raça canina não interfere na motilidade espermática.

A redução de motilidade espermática decorrente do estresse, encontrada neste estudo, também foi um dos efeitos observados em animais de produção submetidos ao estresse térmico ${ }^{3,18,20}$, em ratos submetidos ao estresse psicológico crônico ${ }^{21}$, e em homens sob intenso treinamento físico e expostos a estresse psicológico agudo ${ }^{13}$.

A análise dos dados relativos ao vigor espermático revelou efeito estatístico do tratamento ( $p<0,0001$; Tabela 2). Embora o número de animais utilizados seja considerado adequado para um experimento com cães, ele não foi suficiente para demonstrar o efeito da interação tratamento $\mathrm{X}$ tempo, e somente foi possível observar uma tendência de efeito desta interação $(p=0,0628)$. Devido a isso, procedeu-se à análise dos efeitos da interação tratamento $\mathrm{X}$ tempo onde observou-se efeito estatístico significante nas semanas 1, 4, 7 e 9, cuja carga estressora foi mais intensa: semana 1 (fase 1) - troca de tratador e de rotina; semana 4 (fase 3)- início do adestramento especializado, acrescido de maior carga de exercícios físicos; semana 7 (fase 4) - período de manobras militares, denominado acampamento, com exercícios de tiro real, natação, atividades de ataque e defesa; semana 9 (fase 5) - volta do grupo tratamento à situação de repouso. Estes achados indicam

Tabela 2 - Média e desvio padrão (Md \pm DP) da motilidade progressiva (\%) e do vigor espermáticos (0-5) de cães militares da raça rottweiler submetidos ao estresse de treinamento (GT) e de cães em repouso (GC), em função do tempo (semana) e fase do treinamento - 2001

\begin{tabular}{|c|c|c|c|c|c|}
\hline \multirow{3}{*}{$\begin{array}{c}\text { Fase do } \\
\text { Treinamento }^{1}\end{array}$} & \multirow{3}{*}{ Semana } & \multicolumn{4}{|c|}{ Parâmetro (Md $\pm \mathrm{DP})$} \\
\hline & & \multicolumn{2}{|c|}{ Motilidade Espermática } & \multicolumn{2}{|c|}{ Vigor Espermático } \\
\hline & & GT & GC & GT & GC \\
\hline 1 & 1 & $53,75 \pm 26,32$ & $86,00 \pm 6,36$ & $2,51 \pm 0,72$ & $3,53 \pm 0,45$ \\
\hline \multirow{2}{*}{2} & 2 & $59,68 \pm 28,11$ & $71,00 \pm 19,69$ & $2,43 \pm 0,75$ & $2,77 \pm 0,74$ \\
\hline & 3 & $61,56 \pm 13,02$ & $69,50 \pm 14,85$ & $2,40 \pm 0,53$ & $2,80 \pm 0,43$ \\
\hline \multirow{2}{*}{3} & 4 & $63,28 \pm 12,90$ & $74,75 \pm 6,28$ & $2,48 \pm 0,54$ & $3,42 \pm 0,50$ \\
\hline & 5 & $59,68 \pm 14,41$ & $66,00 \pm 17,28$ & $2,68 \pm 0,51$ & $2,77 \pm 0,53$ \\
\hline \multirow{2}{*}{4} & 6 & $56,25 \pm 10,26$ & $68,75 \pm 8,35$ & $2,50 \pm 0,37$ & $2,82 \pm 0,40$ \\
\hline & 7 & $51,56 \pm 20,17$ & $71,50 \pm 6,25$ & $2,43 \pm 0,60$ & $3,15 \pm 0,24$ \\
\hline \multirow{2}{*}{5} & 8 & $60,93 \pm 13,55$ & $73,50 \pm 5,02$ & $2,68 \pm 0,37$ & $3,07 \pm 0,20$ \\
\hline & 9 & $51,87 \pm 14,31$ & $72,75 \pm 8,93$ & $2,53 \pm 0,45$ & $3,25 \pm 0,44$ \\
\hline \multicolumn{2}{|l|}{ Média } & $57,62 \pm 17,49$ & $72,63 \pm 12,26$ & $2,52 \pm 0,53$ & $3,06 \pm 0,52$ \\
\hline \multicolumn{2}{|l|}{$\mathrm{p}$} & \multicolumn{2}{|c|}{$<0,001$} & \multicolumn{2}{|c|}{$<0,001$} \\
\hline
\end{tabular}

\footnotetext{
${ }^{1}$ Fase do Treinamento: 1 . Adaptação dos cães ao canil e ao manejo; 2 . Exercícios de adestramento básico; 3 . Condicionamento físico e exercícios de adestramento; 4. Exercícios no acampamento (fase de maior trabalho e desgaste físico); 5. Período de repouso dos cães
} 
que o vigor espermático é um parâmetro sensível, e que sua variação é dependente do agente estressor. Embora a nona semana tenha sido denominada "semana sem estresse ou de repouso" para o grupo tratamento, ela provavelmente gerou ansiedade, um estresse psicológico, nos animais, pela ausência de atividades. $\mathrm{O}$ efeito do estresse psicológico sobre os parâmetros seminais já está bem estabelecido em humanos ${ }^{5,13}$.

Verificaram-se também correlações significativas entre a motilidade e vigor espermático e as alterações morfológicas espermáticas. As correlações entre o vigor espermático e os defeitos maiores $(\mathrm{r}=-0,438$; $\mathrm{p}<0,0001)$, defeitos menores $(r=-0,217$; $\mathrm{p}=0,044)$ e defeitos totais $(\mathrm{r}=-0,415$; $\mathrm{p}<0,0001)$; e entre a motilidade espermática e os defeitos maiores $(\mathrm{r}=-0,458 ; \mathrm{p}<0,0001)$ e defeitos totais $(\mathrm{r}=-0,409 ; \mathrm{p}<0,0001)$, demonstram uma estreita relação entre alterações morfológicas espermáticas, motilidade e vigor espermático.

O estresse diminui a ingestão de água, interferindo na homeostase', que por sua vez poderia interferir no $\mathrm{pH}$ seminal. Todavia não se observou este fato neste experimento já que o pH seminal dos animais GC e GT não diferiu $(p>0,05)$ entre si.

A concentração espermática dos animais GT e GC corroboram os achados da literatura ${ }^{11,12,22}$. Os resultados deste experimento demonstraram que o estresse não interferiu na concentração espermática, o que está em discordância com os achados de Fukuda et al. ${ }^{13}$. Tal discordância pode ser decorrente do agente estressor avaliado.

Não houve efeito estatístico do tratamento sobre a porcentagem de defeitos menores, contrariando Coubrough ${ }^{3}$ que relatou que as patologias espermáticas secundárias ou defeitos de morfologia espermática menores, indicativos de mau funcionamento epididimário, são influenciados por agentes estressantes.

Com relação à porcentagem de defeitos morfológicos menores, os dados obtidos neste trabalho encontram-se dentro do limite de normalidade ${ }^{22}$. Entretanto Leite $\mathrm{Netto}^{14}$, que utilizou cães da raça Boxer, encontrou valores inferiores, e Souza et al. ${ }^{12}$, que avaliou animais da raça Pastor alemão, registrou valores superiores. Tais resultados sugerem que este parâmetro pode ser influenciado pela variável raça.

Os defeitos maiores servem como indicativo de alterações ocorridas durante a produção espermática ${ }^{6,14}$. Quando em número elevado, caracterizam um sêmen de baixa fertilidade, mesmo que certos tipos de anormalidades possam não estar associadas à infertilidade ${ }^{22}$. $\mathrm{O}$ estresse aumenta de modo significativo $(\mathrm{p}<0,0001)$ a porcentagem de defeitos maiores (Tabela 3). No presente trabalho, o aumento na porcentagem de defeitos maiores deveu-se principalmente à ocorrência de cabeças anormais soltas e caudas fortemente dobradas e enroladas. Entretanto, não foi possível demonstrar o efeito estatístico do tipo de fonte estressora sobre este parâmetro (interação tratamento $\mathrm{X}$ tempo).

Houve efeito significativo $(\mathrm{p}<0,001)$ do estresse sobre os defeitos totais. Em touros $^{18}$ e em humanos ${ }^{5,13}$ verificou-se um aumentou significativamente $\mathrm{da}$ porcentagem de alterações morfológicas decorrentes do estresse. Todavia, neste estudo, não foram constatados efeitos da interação tempo $\mathrm{X}$ tratamento sobre a porcentagem de defeitos morfológicos totais, indicando que neste parâmetro a intensidade de alterações encontradas não está relacionada ao tipo de agente estressor. Os cães do GC apresentaram valores dentro do limite de normalidade ${ }^{11,22}$.

Giblin et al..$^{5}$ observaram correlação negativa entre o estresse e a porcentagem de espermatozóides morfologicamente normais em humanos. Infelizmente tal correlação entre defeitos morfológicos totais e níveis plasmáticos de cortisol, não pôde ser evidenciada neste experimento.

\section{Conclusões}

O presente estudo conclui que o treinamento militar representa uma fonte de estresse para cães machos da raça Rottweiller, interfere nos níveis plasmáticos de cortisol e 
Tabela 3 - Média e desvio padrão (Md \pm DP) da porcentagem (\%) de defeitos maiores e defeitos menores de cães militares da raça rottweiler submetidos ao estresse de treinamento (GT), e de cães em repouso (GC), em função do tempo (semanas) e da fase do treinamento - 2001

\begin{tabular}{|c|c|c|c|c|c|}
\hline \multirow{3}{*}{$\begin{array}{c}\text { Fase do } \\
\text { Treinamento }^{1}\end{array}$} & \multirow{3}{*}{ Semana } & \multicolumn{4}{|c|}{ Parâmetro (Md $\pm \mathrm{DP})$} \\
\hline & & \multicolumn{2}{|c|}{ Defeitos Maiores } & \multicolumn{2}{|c|}{ Defeitos Menores } \\
\hline & & GT & GC & GT & GC \\
\hline 1 & 1 & $31,85 \pm 26,11$ & $17,08 \pm 5,09$ & $46,26 \pm 35,05$ & $35,21 \pm 21,14$ \\
\hline \multirow{2}{*}{2} & 2 & $28,49 \pm 13,55$ & $16,69 \pm 7,16$ & $42,56 \pm 20,18$ & $30,97 \pm 16,46$ \\
\hline & 3 & $26,24 \pm 18,60$ & $14,96 \pm 9,01$ & $36,58 \pm 23,39$ & $28,86 \pm 11,28$ \\
\hline \multirow{2}{*}{3} & 4 & $24,38 \pm 10,97$ & $12,30 \pm 8,02$ & $37,66 \pm 12,1$ & $24,86 \pm 9,25$ \\
\hline & 5 & $26,77 \pm 10,29$ & $15,75 \pm 8,96$ & $47,23 \pm 16,42$ & $27,16 \pm 12,80$ \\
\hline \multirow{2}{*}{4} & 6 & $24,84 \pm 9,30$ & $13,51 \pm 6,31$ & $36,87 \pm 12,48$ & $25,62 \pm 10,51$ \\
\hline & 7 & $35,32 \pm 20,69$ & $15,69 \pm 11,83$ & $51,50 \pm 25,48$ & $29,62 \pm 12,63$ \\
\hline \multirow{2}{*}{5} & 8 & $20,27 \pm 7,76$ & $18,32 \pm 1,63$ & $28,64 \pm 8,10$ & $32,56 \pm 27,12$ \\
\hline & 9 & $23,76 \pm 8,66$ & $21,62 \pm 17,33$ & $36,74 \pm 10,53$ & $32,76 \pm 23,31$ \\
\hline \multicolumn{2}{|c|}{ Média } & $26,80 \pm 14,90$ & $16,01 \pm 11,17$ & $40,34 \pm 19,92$ & $29,61 \pm 11,17$ \\
\hline \multicolumn{2}{|l|}{$\mathrm{p}$} & \multicolumn{2}{|c|}{$<0,0001$} & \multicolumn{2}{|c|}{$<0.0001$} \\
\hline
\end{tabular}

1 Fase do Treinamento: 1. Adaptação dos cães ao canil e ao manejo; 2. Exercícios de adestramento básico; 3 . Condicionamento físico e exercícios de adestramento; 4. Exercícios no acampamento (fase de maior trabalho e desgaste físico); 5. Período de repouso dos cães

afeta algumas características seminais, como motilidade progressiva, vigor espermático, defeitos morfológicos espermáticos maiores e defeitos morfológicos espermáticos totais.

\section{Evaluation of work stress effects on Rottweilers male dog's fertility}

\section{Abstract}

The present work aimed to evaluate the effect of work stress male dogs fertility. Eighteen fertile male Rottweiler dogs, mean aged 4 years old, were randomly allocate into two groups: resting (control) and working (treatment). Treatment consisted of 5 working stages: 1 - adaptation, 2- basic obedience training, 3 - physical conditioning and military training, 4 - camping and 5 - resting. During the experimental period, semen was collected and evaluated twice a week. At the end of each stage, blood collections were performed in order to evaluate plasmatic levels of cortisol. Data were analyzed using the statistical software SAS ( $a=5 \%$ ). A significant negative effect of stress was observed on sperm motility (resting $=72.63$ vs. working $=57.62$, $\mathrm{p}<0.001$ ), progressive motility (resting $=3.06$ vs. working $=2.52$, $\mathrm{p}<0.0001$ ), major defects (resting $=16.01$ vs. working $=26.80$, $\mathrm{p}<0.0001$ ) and total defects (resting $=29.61$ vs. working $=40.34$, $\mathrm{p}<0.0001)$. Furthermore, a significant interaction $(\mathrm{P}<0.0001)$ was observed between treatment and working stage periods for the plasmatic levels of cortisol, suggesting that the level of stress may vary according to the type of work. Results indicated that work stress may negatively influence fertility in male dogs.
Key words: Semen. Stress. Dogs. 


\section{Referências}

1 CASE, L. P.; CAREY, D. P.; HIRAKAWA, D. A. Atividades e estresse. In: CASE, L. P.; CAREY, D. P.; HIRAKAWA, D. A. Nutrição canina e felina. Madrid: Harcourt Brace de España, 1998. p. 219-226.

2 ENCARNAÇÃO, R. E. Estresse e produção animal. Campo Grande: EMBRAPA-CNPGC, 1997. 32 p.

3 COUBROUGH, R. I. Stress and fertility: a review. Onderstepoort Journal of Veterinary Research, v. 52 n. 3, p. 153-156, 1985.

4 DOBSON, H.; SMITH, R. F. Stress and reproduction in farm animals. Journal of Reproduction and Fertility, v. 49 , p. 451-461, 1995. Supplement.

5 GIBLIN, P. T.; POLAND, M. L.; MOGHISSI, K. S.; AGER, J. W.; OLSON, J. M. Effects of stress and characteristics adaptability on semen quality in healthy men. Fertility and Sterility, v. 49, n. 1, p. 127-132, 1988.

6 VANNUCCHI, C. I.; SATZINGER, S.; SANTOS, S. E. C. Avaliação seminal em cães- aspectos práticos. Clínica Veterinária, v. 3, n. 15, p. 22-27, 1998.

7 NATIONAL RESEARCH COUNCIL. Nutrient requirements of dogs. Washington: National Academy Press, 1985. 78 p.

8 BEERDA, B.; SCHILDER, M. B. H.; VAN HOOF, J. A. R. A. M.; DE VRIES, H. W. Manifestations of chronic and acute stress in dogs. Applied Animal Behaviour Science, v. 52, p. 307-319, 1997.

9 CAMACHO, A. A. Determinação dos níveis séricos de cortisol pelo método de radioimunoensaio em cães clinicamente normais. 1982. 22 f. Dissertação (Mestrado em Medicina Veterinária) - Escola de Veterinária, Universidade Federal de Minas Gerais, Belo Horizonte, 1982

10 HAND, M. S.; THATCHER, C. D.; REMILLARD, R. L.; ROUDEBUSH, P. Small animal clinical nutrition. 4. ed. Topeka: Mark Morris Institute, 2000. 1192 p.

11 JOHNSTON, S. D. Performing a complete canine semen evaluation in a small animal hospital. Veterinary Clinics of North America: Small Animal Practice, v. 21, n. 9, p. 545-551, 1991.

12 SOUZA, J. A. T.; SPICCIATI, W.; VISINTIN, J. A.; BARNABE, V. H.; BARNABE, R. C. Características seminais de cães da raça Pastor Alemão. Brazilian Journal of Veterinary Research and Animal Science, v. 32, n. 3, p. 181-186, 1995.

13 FUKUDA, M.; FUKUDA, K.; SHIMIZU, T.; YOMURA, W.; SHIMIZU, S. Kobe earthquake and reduced sperm motility. Human Reproduction, v. 11, p.1244-1246, 1996.

14 LEITE NETTO, M. C. Avaliação das características seminais de cães da raça boxer submetidos à suplementação com vitamina E e levedo de cerveja. 2001. 29 f. Dissertação (Mestrado em Reprodução Animal) - Faculdade de Medicina Veterinária e Zootecnia da Universidade de São Paulo, São Paulo, 2001.

15 SEAGER, S. W. J.; PLATZ, C. C. Collection and evaluation of canine semen. Veterinary Clinics of North America, v. 7, p. 757-764, 1977.

16 OETTLÉ, E. E.; SOLEY, T. Sperm abnormalities in the dog: a light and electron microscopic study. Veterinary Medicine Reviews, v. 59, p. 28-70, 1986.

17 BEERDA, B.; SCHILDER, M. B. H.; BERNARDINA, W.; VAN HOOF, J. A. R. A. M.; DE VRIES, H. W.; $M O L, J . A$. Chronic stress in dogs subjected to social and spatial restriction: II - Hormonal and immunological responses. Phisiology \& Behavior, v. 66, n. 2, p. 243254, 1999.

18 BARTH, A. D. Insights to the pathogenesis of sperm abnormalities in bulls. Revista Brasileira de Reprodução Animal, v. 4, p. 1-14, 1993. Suplemento.

19 DAHLBOM, M.; ANDERSSON, M.; HUSZENICZA, G.; ALANKO, M. Poor semen quality in Irish wolfhounds: a clinical, hormonal and spermatological study. Journal of Small Animal Practice, v. 36, p. 547552, 1995.

20 MÜLLER, P. B. Bioclimatologia aplicada aos animais domésticos. 3. ed. Porto Alegre: Sulina, 1989. p. 144217.

21 SUÁREZ, M.; FIOL DE CUNEO, M.; VICENTI, L.; RUIZ, R. D. Changes in cortiscosterone levels and sperm functional activity by chronic stress in rats. Archives of Physiology and Biochemistry, v. 104, n. 3, p. 351-356, 1996.

22 HAFEZ, E. S. E.; HAFEZ, B. Reproduction in farm animals. 7. ed. Philadelphia: Lippincot Williams \& Wilkins, 2000. 